\section{Prevalência de fragilidade e fatores associados em idosos comunitários de Belo Horizonte, Minas Gerais, Brasil: dados do Estudo FIBRA}

\author{
Prevalence of frailty and associated factors in \\ community-dwelling elderly in Belo Horizonte, \\ Minas Gerais State, Brazil: data from the \\ FIBRA study
}

\section{Prevalencia de la fragilidad y factores asociados en ancianos de una comunidad de Belo Horizonte, Minas Gerais, Brasil: datos del Estudio FIBRA}

\begin{abstract}
The prevalence of frailty varies greatly and has received insufficient attention in developing countries. This study aimed to identify the prevalence of frailty and associated factors among the elderly in Belo Horizonte, Minas Gerais State, Brazil. Clinical, functional, and socio-demographic factors were analyzed. An ordinal regression model was used to verify conditions associated with frailty and to determine odds ratios $(\alpha=0.05)$. Prevalence rates were $46.3 \%$ for prefrailty was $8.7 \%$ for weakness. Pre-frail and frail elderly, respectively, showed higher and increasing odds ratios for dependency in instrumental activities of daily living; restrictions in advanced activities of daily living; use of walking aids; comorbidities; falls; depressive symptoms; lower self-efficacy in preventing falls; hospitalization; and advanced age. The study identified a high percentage of frail states associated with higher odds of adverse health conditions, especially related to disability.
\end{abstract}

Frail Elderly; Activities of Daily Living; Prevalence
Renata Alvarenga Vieira 1,2 Ricardo Oliveira Guerra 3 Karla Cristina Giacomin 4 Karina Simone de Souza Vasconcelos 2 Amanda Cristina de Souza Andrade 4 Leani Souza Máximo Pereira 5 João Marcos Domingues Dias 5 Rosângela Corrêa Dias 5

\section{Resumo}

A fragilidade apresenta elevada variabilidade na prevalência e é pouco investigada em países em desenvolvimento. O objetivo deste estudo foi identificar a prevalência e fatores associados à fragilidade em idosos comunitários de Belo Horizonte, Minas Gerais, Brasil. Aspectos clínicos, funcionais e sociodemográficos foram avaliados. Foi utilizado o modelo de regressão ordinal, para verificar condições associadas à fragilidade e determinar a razão de chance $(\alpha=$ 0,05). A prevalência de pré-fragilidade foi $46,3 \%$ e de fragilidade de 8,7\%. Os idosos pré-frágeis e frágeis apresentaram, respectivamente, maiores e crescentes razão de chances para dependência em atividades instrumentais de vida diária; restrição em atividades avançadas de vida diária; utilização de dispositivos auxiliares da marcha; comorbidades; quedas; sintomas depressivos; menor autoeficácia para prevenir quedas; hospitalização e idade avançada. Foi identificado elevado percentual de estados de fragilidade associados a maiores chances para condições adversas de saúde, especialmente as relacionadas à incapacidade.

Idoso Fragilizado; Atividades Cotidianas; Prevalência 


\section{Introd ução}

O termo fragilidade destaca-se nas últimas décadas em estudos sobre o envelhecimento. Apesar de amplamente utilizado, ainda não há uma definição consensual, caracteriza-se como um conceito em evolução que apresenta uma pluralidade de definições operacionais e conceitos, fundamentados em distintos modelos teóricos, o que aumenta a complexidade na compreensão do processo de fragilização 1,2,3,4.

Dentre os referenciais teóricos, o modelo biomédico fundamenta o conceito de fragilidade física como uma síndrome de natureza clínica, multifatorial, caracterizada pelo aumento da vulnerabilidade a estressores, que resulta na diminuição de reservas fisiológicas e desequilíbrio de múltiplos sistemas. Esta definição é embasada por uma tríade de alterações relacionadas ao envelhecimento: sarcopenia, desregulação neuroendócrina e disfunção imunológica 1,2,5,6.

A adoção desse referencial possibilitou um avanço relacionado à operacionalização dos critérios diagnósticos de fragilidade, por meio da aplicabilidade do fenótipo de fragilidade proposto por Fried et al. 1, com base nos dados do Cardiovascular Health Study. A presença de três ou mais de cinco componentes clínicos - perda de peso não intencional, fraqueza muscular, fadiga, baixo nível de atividade física e diminuição da velocidade de marcha 1 - caracteriza a fragilidade. Tal fenótipo apresenta validade preditiva e ampla utilização em estudos de base populacional, pois permite identificar o processo de transição entre saúde e fragilidade, funcionando como ferramenta clínica e de rastreio 1,5,6.

A fragilidade prediz desfechos adversos à saúde, tais como: agravamento de doenças, comorbidades, quedas, institucionalização, hospitalização, incapacidade e morte 1,2,6,7. Essa síndrome tem impacto negativo sobre o processo de envelhecimento, favorecendo a coexistência dele com a incapacidade, assim como o aumento da utilização e custos de serviços de saúde 6,8.

A fragilidade associa-se à idade avançada 1,9,10,11,12, as condições socioeconômicas e de saúde desfavoráveis como renda insuficiente, baixo nível educacional, percepção negativa de saúde, incapacidade funcional, institucionalização, quedas, presença de comorbidades, doenças agudas e hospitalização em populações idosas de diversos países e no Brasil 1,2,6,7,10,11,12.

Na Europa e na América do Norte, a prevalência de fragilidade varia de 5,8\% a 27,3\%, sendo maior entre as mulheres, idosos institucionalizados e com idade avançada 1,9,10. Contudo, em países em desenvolvimento, nos quais o processo de envelhecimento emerge em condições de saúde, econômicas e sociais, frequentemente desfavoráveis, foram observadas prevalências de fragilidade com tendências mais elevadas de $26,7 \%$ a $42,6 \%$ 11,12. Portanto, o processo de fragilização dessas populações necessita de investigação aprofundada, para identificar os fatores passíveis de prevenção e intervenção associados à síndrome nessas populações.

Este estudo teve o propósito de determinar os pontos de corte para o fenótipo de fragilidade, verificar a prevalência e os fatores associados a essa síndrome em idosos comunitários de Belo Horizonte, Minas Gerais, Brasil, tendo em conta a ordem de gravidade entre os estados de préfragilidade e fragilidade.

\section{Materiais e método}

Trata-se de um estudo transversal de base populacional, aprovado pelo Comitê de Ética em Pesquisa da Universidade Federal de Minas Gerais, parecer no ETIC 187/07, integrado à Rede FIBRA (Rede de Estudos de Fragilidade em Idosos Brasileiros). Rede multicêntrica que avaliou 7 mil idosos residentes em 17 cidades das cinco regiões brasileiras para determinar a prevalência e fatores associados à fragilidade no Brasil.

O estudo se baseou em uma amostra probabilística de 601 idosos comunitários residentes em Belo Horizonte, acrescida de $30 \%$ prevendo possíveis perdas e recusas. Estipulou-se o valor de $4 \%$ de erro amostral para determinar maior precisão nas estimativas de municípios com mais de 1 milhão de habitantes. Foi realizada amostragem estratificada segundo a densidade populacional de idosos (baixa, média e alta), baseada nos dados do Censo Demográfico de 2000 (Instituto Brasileiro de Geografia e Estatística. http:// www.ibge.gov.br). Em cada estrato foram selecionados aleatoriamente cinco setores censitários, nos quais foram percorridos todos os domicílios, perfazendo um total de 15 setores. Os idosos foram avaliados de dezembro de 2008 a setembro de 2009, em uma única visita domiciliar. O efeito da estratificação amostral foi testado, mas não foi significativo para o cálculo das prevalências ou estimativas de fragilidade.

\section{Critérios de inclusão e exclusão}

Todos os idosos de ambos os sexos, residentes nos setores censitários aleatorizados que tivessem 65 anos ou mais e $\geq 17$ pontos no Mini-Exame do Estado Mental (MEEM) 13, foram convidados a participar. Foram excluídos do estudo os indivíduos que, além do déficit cognitivo, tivessem doenças ou sequelas que impedissem a realiza- 
ção dos testes (déficits graves motores, visuais, auditivos e afasia), usuários de cadeira de rodas e idosos acamados ou com doenças em estágio terminal.

Nos 15 setores censitários selecionados, os examinadores abordaram 1.640 domicílios. Desses, em 427 não houve resposta, em 436 não havia idosos e 86 idosos se recusaram a participar da pesquisa. Dos 685 domicílios onde pelo menos um idoso concordou em participar, 47 participantes foram excluídos por não atingirem a pontuação mínima no MEEM, dez tinham idade $<65$ anos, sete foram excluídos por imobilidade, demência ou doenças graves, seis interromperam a entrevista, 12 não completaram todos os testes e dois foram excluídos por terem sido avaliados duas vezes.

\section{Instrumentos e variáveis}

A variável dependente do estudo, síndrome da fragilidade, foi operacionalizada segundo o fenótipo proposto por Fried et al. 1: (1) perda de peso avaliada pelo autorrelato, indicando resposta positiva à perda $\geq \mathrm{a} 4,5 \mathrm{~kg}$ ou $\geq 5 \%$ do peso corporal; (2) exaustão avaliada por duas perguntas da escala de depressão do Center for Epidemiological Studies (CES-D) 14; preenchendo o critério caso ocorresse resposta positiva em pelo menos uma delas; (3) nível de atividade física investigado pelo questionário Minnesota Leisure Time Physical Activity Questionnaire (MLTPAQ) 15,16, apresentado como medida o gasto calórico semanal em quilocalorias (Kcal), ajustado por sexo e estabelecido como pontos de corte o percentil 20 da amostra (idosos abaixo do ponto de corte pontuariam nesse critério); (4) força muscular avaliada pelo teste de força de preensão palmar, ajustado por sexo e quartis do índice de massa corporal (IMC) com pontos de corte determinados pelo percentil 20 da distribuição, utilizando o dinamômetro do tipo Jamar (Sammons Preston, Warrenville, Estados Unidos); (indivíduos abaixo do ponto de corte pontuariam neste critério); (5) tempo de marcha calculado em segundos, medido pelo cronômetro Professional Quartz Timer, modelo KD1O69 (Kadio, China), gasto para percorrer em velocidade habitual, uma distância de 4,6 metros, de um total de 8,6 metros considerando 2,0 metros para aceleração e 2,0 metros para desaceleração da marcha (os pontos de corte da amostra foram determinados pelo percentil 80 do tempo, ajustado por sexo e altura; indivíduos acima do ponto de corte preencheriam este critério).

Os pontos de corte dos componentes 3, $4 \mathrm{e}$ 5 foram determinados para a amostra do estudo. De acordo com a pontuação nos cinco componentes, os idosos foram considerados frágeis quando preenchiam três ou mais critérios, pré-frágeis na presença de um ou dois critérios e não frágeis na ausência dos critérios.

As variáveis independentes do estudo foram avaliadas por inquérito multidimensional padronizado pela Rede FIBRA no qual foram agrupadas em blocos investigativos: que avaliaram aspectos sociodemográficos, econômicos e de condição de saúde física, mental e funcional. Para o presente trabalho foram selecionados: (a) aspectos sociodemográficos e econômicos: sexo, idade, escolaridade, estado civil, arranjo familiar, raça, ocupação atual, considerar a renda suficiente, responsabilidade pelo sustento familiar e possuir residência própria; (b) condição de saúde e hábitos de vida: índice de comorbidades conforme o autorrelato de diagnóstico médico de doenças crônicas no último ano (doença cardíaca, hipertensão arterial, acidente vascular cerebral - AVC, diabetes mellitus, câncer, artrite, doença respiratória, depressão e osteoporose); e autorrelato dos seguintes eventos: restrição ao leito, uso de dispositivos auxiliares da marcha (bengala, muletas e andadores), dificuldade de memória, dificuldade para dormir, quedas nos últimos 12 meses, número de medicamentos utilizados, déficit visual e auditivo, tabagismo e alcoolismo; (c) incapacidade funcional autorrelatada: conforme questionário para verificar a realização de atividades avançadas de vida diária (AAVD) e aplicação das escalas Katz-Brasil 17 para atividades básicas de vida diária (ABVD) e Lawton para atividades instrumentais de vida diária (AIVD) 18; (d) utilização e cobertura de serviços de saúde: frequência nos últimos 12 meses de hospitalização, consultas médicas e atendimentos domiciliares. Tipo de cobertura nos atendimentos de saúde (público/privado); (e) cuidado e expectativa de cuidado em atividades de vida diária (AVD) incorporando as AAVD, AIVD e ABVD, avaliadapor duas questões: " $O(A) \operatorname{senhor}(a)$ tem algum parente, amigo ou vizinho que poderia cuidar de você por alguns dias, caso necessário?" e "Caso precise ou venha precisar de ajuda para realizar qualquer destas atividades o(a) senhor(a) tem com quem contar?"; (f) autoeficácia para prevenir quedas: medida pela versão brasileira da Falls Efficacy Scale-International (FES-I Brasil) 19 que avalia a preocupação com a possibilidade de cair ao realizar 16 atividades rotineiras em uma escala contínua de 0 a 64 pontos, sem pontos de corte; (g) sintomas depressivos: avaliados pela Escala de Depressão Geriátrica (EDG-15) que avalia o estado socioafetivo do idoso, com domínios que compreendem humor, sintomas somáticos, interações com outros indivíduos e funcionamento motor com ponto de corte indicativo de sintomas depressivos igual ao escore de seis pon- 
tos 20; (h) avaliação subjetiva de saúde: avaliada pela perguta: "Em geral o(a) senhor(a) diria que sua saúde é: muito boa; boa; regular; ruim ou muito ruim?"; (i) satisfação global com a vida: avaliada pela pergunta: “ $O(a)$ senhor(a) está satisfeito com sua vida hoje?", apresentando as seguintes categorias de resposta: pouco, mais ou menos e muito satisfeito.

\section{Análise estatística}

Foi realizada a análise descritiva com medidas de tendência central para a caracterização da amostra. Análise univariada foi utilizada para verificar a associação entre fragilidade e as variáveis independentes com utilização dos testes qui-quadrado de Pearson, exato de Fisher e Kruskal-Wallis. As variáveis com valor de $\mathrm{p}$ menor que 0,20 foram incluídas no modelo de regressão multivariada.

$\mathrm{Na}$ análise multivariada, ajustou-se um modelo de regressão logística ordinal estereótipo (ME) que pode ser considerado uma extensão do modelo multinomial e compara cada categoria da variável-resposta com uma categoria de referência 21 .O ME deve ser utilizado quando a variável resposta é uma variável ordinal com categorias discretas, como é o caso da fragilidade.

$\mathrm{O} \mathrm{ME}$ atribui pesos aos coeficientes do modelo, representando o caráter ordinal da variável resposta. Esses pesos são diretamente relacionados ao efeito das covariáveis. Portanto, a odds ratio formada tem uma tendência crescente, pois os pesos normalmente são construídos com ordenação 21. Para verificar a adequação do modelo, utilizou-se o teste da deviance. Foram utilizados os softwares SPSS 15.0 (SPSS Inc., Chicago, Estados Unidos) e Stata 10.0 (Stata Corp., College Station, Estados Unidos).

\section{Resultados}

Amostra de 601 idosos comunitários, com média etária de 74,3 $( \pm 6,43)$ anos, constituída por maioria de mulheres 398 (66,2\%), com maior percentual de escolaridade 356 (59,2\%) entre zero e quatro anos de estudo, situação conjugal predominante em $311(51,7 \%)$ idosos sem parceiros, $518(86,2 \%)$ não moravam sozinhos, 311 $(51,9 \%)$ com maior frequência de raça não branca, maioria de $447(74,4 \%)$ aposentados, com utilização de plano privado de saúde em 373 $(62,1 \%)$ dos indivíduos, $344(57,3 \%)$ da amostra avaliaram sua renda suficiente para as necessidades diárias.

A distribuição dos itens positivos para a composição do fenótipo de fragilidade nessa amostra revelou uma proporção de $52(8,7 \%)$ de indivíduos frágeis; 278 (46,3\%) de pré-frágeis e 271 (45,0\%) de idosos não frágeis.

Os pontos de corte nos componentes do fenótipo de fragilidade utilizados para determinar a prevalência dessa síndrome na população idosa comunitária de Belo Horizonte foram estabelecidos tendo em vista as medidas determinadas na amostra representativa desse município, a saber: a) O percentil 20 da força muscular (ajustado por sexo e IMC): para as mulheres (IMC $\leq 24,33$ $=14 \mathrm{~kg}$; IMC de 24,34 a 27,34 = 14,13kg; IMC de $27,35$ a $31,07=14,33 \mathrm{~kg} ;$ IMC $>31,08=14,33 \mathrm{~kg})$, para os homens $(\mathrm{IMC} \leq 22,89=19,60 \mathrm{~kg}$; IMC entre 22,90 e 25,01 = 22,93kg; IMC entre 25,02 e $28,35=22 \mathrm{~kg}$; IMC > 28,36 = 25kg);

b) O percentil 80 do tempo de marcha para mulheres com estaturas $\leq 1,53 \mathrm{~m}$ foi $\geq 7,15$ segundos $\mathrm{e}$ para aquelas com altura $>1,53 \mathrm{~m}$ foi $\geq 6,65$ segundos. Para os homens com estatura $\leq 1,67 \mathrm{~m}$ foi $\geq$ 5,73 segundos e aqueles com altura $>1,67 \mathrm{~m}$ foi $\geq$ 5,75 segundos;

c) O percentil 20 de gasto calórico para as mulheres foi de $<123 \mathrm{Kcal}$ e para os homens de $<$ $50 \mathrm{Kcal}$.

As frequências dos itens do fenótipo de fragilidade presentes na amostra são apresentadas na Tabela 1. Entre os idosos pré-frágeis e frágeis, os componentes do fenótipo com maior frequência foram, respectivamente, o reduzido nível de atividades físicas e a lentidão de marcha, e, em ambos os grupos, o componente com menor frequência foi a fadiga.

Nos resultados da análise univariada, serão reportados dentre os blocos de variáveis independentes selecionadas para o estudo as variáveis que apresentaram valor de $\mathrm{p}<0,20$ e foram introduzidas no modelo multivariado.

A Tabela 2 apresenta a distribuição das variáveis físico-funcionais, percepção subjetiva de saúde e satisfação com a vida. Houve diferença estatisticamente significativa para as medidas de necessidade de ajuda em ABVD, dependência em AIVD e incapacidade para realizar AAVD. Observaram-se maiores escores para a escala AAVD à medida que aumentava o grau da fragilidade, enquanto para a escala ABVD a direção foi inversa: os escores eram menores à medida que aumentava a fragilidade. Também houve aumento no 3o quartil da escala AIVD entre os indivíduos classificados como frágeis. Ocorreu associação significativa entre a fragilidade e percepção de saúde, com percentual crescente de percepção negativa de saúde dos indivíduos saudáveis para os pré-frágeis e desses para os frágeis. A medida de satisfação global com a vida apresentou associação significativa com fragilidade, com gradiente de satisfação negativa entre os grupos de idosos não frágeis, pré-frágeis e frágeis. 
Tabela 1

Distribuição de frequência dos itens do fenótipo de fragilidade, na amostra de idosos do Município de Belo Horizonte, Minas

Gerais, Brasil.

\begin{tabular}{|c|c|c|c|c|}
\hline \multirow[t]{3}{*}{ Itens do fenótipo } & \multicolumn{3}{|c|}{ Fragilidade } & \multirow{3}{*}{$\begin{array}{l}\text { Total } \\
\text { n (\%) }\end{array}$} \\
\hline & Não frágil & Pré-frágil & Frágil & \\
\hline & n (\%) & n (\%) & n (\%) & \\
\hline \multicolumn{5}{|l|}{ Perda de peso } \\
\hline Não & $271(100,0)$ & $201(72,3)$ & $24(46,2)$ & $496(82,5)$ \\
\hline Sim & $0(0,0)$ & $77(27,7)$ & $28(53,8)$ & $105(17,5)$ \\
\hline \multicolumn{5}{|l|}{ Fraqueza muscular } \\
\hline Não & $271(100,0)$ & $210(75,5)$ & $12(23,1)$ & $493(82,0)$ \\
\hline Sim & $0(0,0)$ & $68(24,5)$ & $40(76,9)$ & $108(18,0)$ \\
\hline \multicolumn{5}{|l|}{ Fadiga } \\
\hline Não & $271(100,0)$ & $217(78,1)$ & $28(53,8)$ & $516(85,9)$ \\
\hline Sim & $0(0,0)$ & $61(21,9)$ & $24(46,2)$ & $85(14,1)$ \\
\hline \multicolumn{5}{|l|}{ Lentidão na marcha } \\
\hline Não & $271(100,0)$ & $204(73,4)$ & $7(13,5)$ & $482(80,2)$ \\
\hline Sim & $0(0,0)$ & $74(26,6)$ & $45(86,5)$ & $119(19,8)$ \\
\hline \multicolumn{5}{|c|}{ Baixo nível de atividade física } \\
\hline Não & $271(100,0)$ & $193(69,4)$ & $18(34,6)$ & $482(80,2)$ \\
\hline Sim & $0(0,0)$ & $85(30,6)$ & $34(65,4)$ & $119(19,8)$ \\
\hline
\end{tabular}

Tabela 2

Distribuição das variáveis físico-funcionais, percepção de saúde e satisfação com a vida, conforme o fenótipo da fragilidade, na amostra de idosos do Município de Belo Horizonte, Minas Gerais, Brasil.

\begin{tabular}{|c|c|c|c|c|c|}
\hline & \multicolumn{3}{|c|}{ Fragilidade } & \multirow[t]{2}{*}{ Total } & \multirow{3}{*}{$\begin{array}{l}\text { Valor } \\
\text { de } p\end{array}$} \\
\hline & Não frágil & Pré-frágil & Frágil & & \\
\hline & Mediana (percentil 25-75) & Mediana (percentil 25-75) & Mediana (percentil 25-75) & Mediana (percentil 25-75) & \\
\hline$A B V D$ & $21,0(21,0-1,0)$ & $21,0(19,00-21,00)$ & $17,0(14,3-19,8)$ & $21,0(20,0-21,0)$ & $<0,001$ * \\
\hline AIVD & $0,0(0,0-0,0)$ & $0,0(0,0-0,0)$ & $0,0(0,0-1,0)$ & $0,0(0,0-0,0)$ & $<0,001$ * \\
\hline \multirow[t]{2}{*}{ AAVD } & $3,0(2,0-4,0)$ & $4,0(2,0-6,0)$ & $5,0(3,0-7,0)$ & $4,0(2,0-5,0)$ & $<0,001$ * \\
\hline & n (\%) & n (\%) & n (\%) & n (\%) & \\
\hline \multicolumn{6}{|l|}{ Percepção de saúde } \\
\hline \multirow[t]{2}{*}{ Muito boa/Boa } & $190(70,1)$ & $148(53,4)$ & $18(34,6)$ & $356(59,3)$ & $<0,001$ \\
\hline & & & & & $\star \star$ \\
\hline Regular/Ruim/Muito & $81(29,9)$ & $129(46,6)$ & $34(65,4)$ & $244(40,7)$ & \\
\hline ruim & & & & & \\
\hline \multicolumn{6}{|l|}{ Satisfação com a vida } \\
\hline Pouco/Mais ou menos & $80(29,5)$ & $113(40,6)$ & $22(42,3)$ & $215(35,8)$ & 0,015 ** \\
\hline Muito & $191(70,5)$ & $165(59,4)$ & $30(57,7)$ & $386(64,2)$ & \\
\hline
\end{tabular}

AAVD: atividades avançadas de vida diária; ABVD: atividades básicas de vida diária; AIVD: atividades instrumentais de vida diária; AVD: atividades de vida diária.

* Teste Kruskall-Wallis;

** Teste de qui-quadrado. 
Os resultados apresentados na Tabela 3 demonstram que existiu associação significativa entre a variável resposta fragilidade e as condições clínicas e adversas de saúde selecionadas. Em todos os casos, nota-se um aumento do número de doenças ou condições adversas à saúde à proporção que a condição de fragilidade se agrava entre os grupos. Também foi demonstrada associação significativa e crescente com o número de medicamentos e a progressão da condição de fragilidade e entre essa e o uso de serviços de saúde. A autoeficácia para realizar atividades rotineiras diante da possibilidade de quedas foi associada à fragilidade, apresentando diminuição progressiva dos indivíduos não frágeis até os frágeis.

No bloco de variáveis sociodemográficas e econômicas, apresentado pela Tabela 4, foi identificada associação significativa entre a fragilidade e pré-fragilidade com idade, estado civil, capacidade de ler e escrever, ocupação, possuir residência própria e ser responsável pelo sustento familiar. Percebe-se aumento gradativo da prevalência da idade avançada, ausência de parceiro e analfabetismo entre os indivíduos não frágeis, pré-frágeis e frágeis. E a redução da prevalência de atividade laboral, possuir residência própria e ser responsável pelo sustento familiar dos idosos não frágeis para os pré-frágeis e desses para os frágeis.

A análise multivariada mostrada na Tabela 5 apresenta as variáveis que permaneceram no modelo após o ajuste pelas variáveis componentes dos blocos investigatórios da análise univariada. O modelo identificou um gradiente positivo de associação entre as variáveis permanentes ao modelo final, conforme a gravidade do fenótipo de fragilidade.

Assim, os idosos frágeis foram aqueles que apresentaram maiores chances em relação aos pré-frágeis e não frágeis para limitações em atividades instrumentais de vida diária, restrição de atividades avançadas, utilização de dispositivos auxiliares da marcha, acometimento por maior quantidade de comorbidades, ocorrência de quedas, sintomas depressivos, redução da autoeficácia, hospitalização e idade avançada.

\section{Discussão}

Na amostra de idosos comunitários do Município de Belo Horizonte, a prevalência de fragilidade foi de $8,7 \%$ e pré-fragilidade de $46,3 \%$. Tais taxas foram semelhantes às verificadas em outros países, por estudos que utilizaram a mesma operacionalização do fenótipo de fragilidade 1,10,22 . Entretanto, esses dados se diferenciam de uma pesquisa brasileira que apontou a prevalência de $17,1 \%$ de fragilidade e $60,1 \%$ de pré-fragilidade, em uma comunidade de Região Nordeste 12. Essas divergências poderiam ser explicadas pelas diferenças entre as populações das regiões Nordeste e Sudeste em contextos sociais e econômicos, captadas por disparidade no Índice de Desenvolvimento Humano (IDH), que desencadeiam impacto sobre as condições de saúde e envelhecimento 23 .

Os critérios diagnósticos de fragilidade determinados pelos pontos de corte dos itens do fenótipo e identificados na população deste estudo foram inferiores aos estabelecidos para o estudo de Fried et al. 1 nos itens referentes: à força muscular, ao tempo de marcha e atividade física. Talvez, os dois primeiros sejam uma particularidade da população brasileira, pois são determinados por medidas antropométricas. Além disso, também foram encontrados valores extremante baixos no domínio atividade física, possivelmente relacionados à inadequação do instrumento MLTPAQ 12,15,16,24 para medir essa variável e/ou à adoção do percentil 20, na população deste estudo, que poderiam ter substimado os valores de gasto calórico, corroborando os achados de Sousa et al. 12 em idosos comunitários da Região Nordeste brasileira. Em estudo de Drey et al. ${ }^{24}$ para idosos europeus, a adequação do MLTPAQ também é questionada por conta da pequena quantidade de atividades do instrumento que são realizadas por idosos, sugerindo inadequação da ferramenta para mensurar o nível de atividade física nessa população. Todavia, a validade da operacionalização do fenótipo proposto por Fried et al. 1 foi amplamente reconhecida em estudos epidemiológicos 1,10,22 e o MLTPAQ foi adaptado para a população brasileira 16 .

Dentre os itens do fenótipo de fragilidade identificados nesta pesquisa, os mais frequentes entre os pré-frágeis foram: a diminuição do peso corporal, a redução da velocidade de marcha e o baixo nível de atividade física. Entre os frágeis, as maiores frequências foram a diminuição de força e a velocidade de marcha. Entretanto, Drey et al. 24 identificaram como mais frequentes entre os pré-frágeis a exaustão e a diminuição da força muscular para idosos comunitários da Alemanha. Enquanto em idosos frágeis os itens mais frequentes do fenótipo, segundo Avila-Furnes et al. 25, em idosos comunitários franceses, foi o baixo nível de atividade física, Ottenbacher et al. 26 identificaram em idosos americanos a redução de velocidade de marcha e a diminuição de força; já Ostir et al. 27 identificaram em idosos americanos de origem mexicana como mais frequentes a redução de velocidade de marcha e a diminuição de força e como menor frequência 
Distribuição de variáveis selecionadas sobre a condição da saúde, utilização de serviços de saúde e consumo de medicamentos conforme o fenótipo da fragilidade, na amostra de idosos do Município de Belo Horizonte, Minas Gerais, Brasil.

\begin{tabular}{|c|c|c|c|c|c|}
\hline & \multicolumn{3}{|c|}{ Fragilidade } & \multirow{3}{*}{$\begin{array}{c}\text { Total } \\
\text { Mediana }\end{array}$} & \multirow[t]{3}{*}{ Valor de $p$} \\
\hline & Não frágil & Pré-frágil & Frágil & & \\
\hline & Mediana & Mediana & Mediana & & \\
\hline Número de doenças crônicas & 1,0 & 2,0 & 2,5 & 1,0 & $<0,001^{\star}$ \\
\hline Número de consultas médicas no último ano & 3,0 & 4,0 & 5,0 & 4,0 & 0,007 * \\
\hline Número de medicamentos & 3,0 & 3,0 & 5,0 & 3,0 & $<0,001$ * \\
\hline \multirow[t]{2}{*}{ FES-I Brasil } & 20,0 & 23,0 & 27,5 & 22,0 & $<0,001$ * \\
\hline & $\mathbf{n}(\%)$ & n (\%) & n (\%) & n (\%) & \\
\hline \multicolumn{6}{|l|}{ Atendimento domiciliar de saúde } \\
\hline Sim & $23(8,5)$ & $40(14,4 \%)$ & $11(21,2)$ & $74(12,3)$ & 0,014 ** \\
\hline \multicolumn{6}{|l|}{ Internação hospitalar } \\
\hline Sim & $28(10,3)$ & $68(24,5)$ & $21(41,2)$ & $117(19,5)$ & $<0,001 * \star$ \\
\hline \multicolumn{6}{|l|}{ EDG-15 (sintomas depressivos) } \\
\hline Sem sintomas depressivos & $233(86,0)$ & $208(74,8)$ & $29(55,8)$ & $470(78,2)$ & $<0,001 * \star$ \\
\hline \multicolumn{6}{|l|}{ Restrição ao leito } \\
\hline $\operatorname{Sim}$ & $22(8,1)$ & $60(21,6)$ & $15(29,4)$ & $97(16,2)$ & $<0,001 * \star$ \\
\hline \multicolumn{6}{|l|}{ Queda } \\
\hline Sim & $53(19,6)$ & $93(33,6)$ & $22(42,3)$ & $168(28,0)$ & $<0,001 * \star$ \\
\hline \multicolumn{6}{|l|}{ Dispositivos de auxilio à marcha } \\
\hline Sim & $6(2,2)$ & $27(9,7)$ & $19(36,5)$ & $52(8,7)$ & $<0,001 * *$ \\
\hline \multicolumn{6}{|l|}{ Dificuldade de memória } \\
\hline $\operatorname{Sim}$ & $117(43,2)$ & $137(49,3)$ & $32(62,7)$ & $286(47,7)$ & $0,028 * \star$ \\
\hline \multicolumn{6}{|l|}{ Alteração sono } \\
\hline Sim & $102(37,6)$ & $130(46,8)$ & $26(50,0)$ & $258(42,9)$ & $0,054 * \star$ \\
\hline
\end{tabular}

EDG-15: Escala de Depressão em Geriatria; FES-I Brasil: Falls Efficacy Scale-International (FES-I Brasil).

* Teste Kruskall-Wallis;

** Teste de qui-quadrado.

a perda de peso e exaustão. A proporção entre os componentes de fragilidade divergem, mesmo entre os estudos com mesma operacionalização, possivelmente por causa das diferenças populacionais $10,12,28$ e pelo fato de a síndrome de fragilidade não se caracterizar como um evento unidirecional, mas de caráter cíclico com vários pontos de desencadeamento do processo e consequente variabilidade nas manifestações iniciais $1,6,12,29,30$.

Sob a ótica da complexidade do modelo teórico que fundamenta a Classificação Internacional de Funcionalidade e Incapacidade (CIF) 31, o conceito de fragilidade adotado no presente estudo prioriza os contextos biológicos e físicos 1,2 Apesar disso, os resultados identificaram a associação entre fragilidade e indicativos de incapacidade funcional, que transpõem a dependência funcional em AVD, o que pode sugerir a presença de componentes vinculados à estrutura e função corporal, atividade, participação social, fatores pessoais e ambientais.

Nessa perspectiva, entre os idosos da amostra de Belo Horizonte, notaram-se maiores chances de fragilidade vinculadas aos componentes da CIF 31 estrutura e função - representados no modelo final, respectivamente, pelo envelhecimento e pela disfunção na marcha. Na mesma direção, compreende-se que a marcha se vincula ao domínio atividade, também contemplado pelo grau de dependência para realizar atividades instrumentais de vida diária; a participação social identificada pela restrição nas atividades avançadas de vida diária; os fatores pessoais assinalados pela limitação da autoeficácia preventiva de quedas, e, finalmente, os ambientais pela hospitalização.

No estudo da fragilidade, o envelhecimento tem sido frequentemente identificado como fator predisponente e como um marco das 
Distribuição das variáveis socioeconômicas e demográficas conforme o fenótipo de fragilidade, na amostra de idosos do Município de Belo Horizonte, Minas Gerais, Brasil.

\begin{tabular}{|c|c|c|c|c|c|}
\hline & \multicolumn{3}{|c|}{ Fragilidade } & \multirow{2}{*}{$\begin{array}{l}\text { Total } \\
\text { n (\%) }\end{array}$} & \multirow[t]{2}{*}{ Valor de $\mathrm{p}$} \\
\hline & $\begin{array}{c}\text { Não frágil } \\
\text { n (\%) }\end{array}$ & $\begin{array}{c}\text { Pré-frágil } \\
\text { n (\%) }\end{array}$ & $\begin{array}{l}\text { Frágil } \\
\text { n (\%) }\end{array}$ & & \\
\hline \multicolumn{6}{|l|}{ Idade (anos) } \\
\hline 80 ou mais & $31(11,4)$ & $57(20,5)$ & $21(40,4)$ & $109(18,1)$ & $<0,001$ * \\
\hline \multicolumn{6}{|l|}{ Estado civil } \\
\hline Sem parceiro & $128(47,2)$ & $149(53,6)$ & $34(65,4)$ & $311(51,7)$ & 0,039 * \\
\hline \multicolumn{6}{|c|}{ Capacidade de ler e escrever } \\
\hline Não & $40(14,8)$ & $56(20,1)$ & $18(36,0)$ & $114(19,0)$ & 0,002 * \\
\hline \multicolumn{6}{|l|}{ Trabalha } \\
\hline Sim & $65(24,0)$ & $38(13,7)$ & $6(11,5)$ & $109(18,1)$ & 0,003 * \\
\hline \multicolumn{6}{|c|}{ Proprietário da residência } \\
\hline $\operatorname{Sim}$ & $226(83,4)$ & $210(75,5)$ & $35(67,3)$ & $471(78,4)$ & $0,011 *$ \\
\hline \multicolumn{6}{|l|}{ Sustenta a família } \\
\hline Sim & $175(65,3)$ & $158(57,2)$ & $28(53,8)$ & $361(60,6)$ & 0,092 * \\
\hline
\end{tabular}

* Teste de qui-quadrado.

Tabela 5

Modelo final de regressão logística ordinal do fenótipo fragilidade e os fatores associados, na amostra de idosos do Município de Belo Horizonte, Minas Gerais, Brasil.

\begin{tabular}{|c|c|c|c|}
\hline Variáveis & Valor de p & $\begin{array}{c}\text { Não frágil x pré-frágil } \\
\text { OR (IC95\%) }\end{array}$ & $\begin{array}{l}\text { Não frágil x frágil } \\
\text { OR (IC95\%) }\end{array}$ \\
\hline \multicolumn{4}{|l|}{ Físico-funcional } \\
\hline AIVD & $<0,001$ & $1,59(1,32-1,90)$ & $2,17(1,48-3,50)$ \\
\hline AAVD & 0,036 & $1,09(1,01-1,18)$ & $1,16(1,01-1,39)$ \\
\hline \multicolumn{4}{|l|}{ Condições de saúde e doenças } \\
\hline Número de doenças crônicas & 0,037 & $1,15(1,01-1,32)$ & $1,27(1,01-1,71)$ \\
\hline Auxílio marcha & 0,031 & $2,36(1,08-5,15)$ & $4,25(1,12-24,75)$ \\
\hline Queda & 0,022 & $1,57(1,07-2,31)$ & $2,14(1,10-5,17)$ \\
\hline \multicolumn{4}{|l|}{ Psicocognitivas } \\
\hline EDG-15 (sintomas depressivos) & 0,008 & $1,77(1,16-2,71)$ & $2,62(1,23-7,02)$ \\
\hline FES-I Brasil & 0,004 & $1,05(1,01-1,08)$ & $1,08(1,02-1,16)$ \\
\hline \multicolumn{4}{|l|}{ Utilização de serviços de saúde } \\
\hline Internação & 0,010 & $1,84(1,16-2,92)$ & $2,78(1,23-8,14)$ \\
\hline \multicolumn{4}{|l|}{ Sociodemográficas } \\
\hline Idade $>80$ anos & 0,032 & $1,65(1,04-2,61)$ & $2,33(1,06-6,55)$ \\
\hline
\end{tabular}

AAVD: atividades avançadas de vida diária; AIVD: atividades instrumentais de vida diária; EDG-15: Escala de Depressão Geriátrica; FES-I Brasil: Falls Efficacy Scale-International (FES-I Brasil).

IC95\%: intervalo de $95 \%$ de confiança; OR: odds ratio.

alterações estruturais desencadeantes do processo de fragilização 1,3,5,6, o que acarreta efeitos sobre o estabelecimento da prevalência de fragilidade e gravidade de sua evolução. No presente estudo, o envelhecimento esteve associado de forma independente à fragilidade, o que condiz com os resultados tanto de pesquisas que caracterizam a fragilidade como o acúmulo de 
déficits ao longo da vida 3,4,32 como de estudos que a consideram uma síndrome de declínios cíclicos em múltiplos sistemas relacionados às condições inerentes ao envelhecimento como sarcopenia, desregulação neuroendócrina e disfunção imunológica 1,5,6,30.

A disfunção na marcha foi verificada de forma independente da operacionalização do fenótipo, pela necessidade de utilização de dispositivos auxiliares representativa dos domínios função e atividade da CIF 31 . A associação entre fragilidade e a utilização de dispositivos auxiliares da marcha constatada neste estudo poderia ser explicada pelos componentes primários da fragilidade - presença de sarcopenia, fadiga e baixo nível de atividade física - por condições subclínicas à síndrome, como quedas e incapacidade 6,33. Portanto, não somente as alterações relacionadas aos componentes primários da fragilidade vinculam-se a lentificação da marcha, assim como a redução da velocidade não constitui um preditor absoluto da utilização de dispositivos de auxílio, visto que os dispositivos podem constituir-se tanto como facilitadores quanto como barreiras à velocidade e à independência funcional na marcha $31,34,35$. Contudo, não foram encontrados estudos que explorassem de modo específico as relações entre o uso de dispositivos auxiliares da marcha e a fragilidade.

A incapacidade ou dependência na realização de atividades de vida diária são frequentemente descritas como representativas do processo de incapacidade em estudos de fragilidade 1,2,5. Neste estudo, elas são consideradas como componentes desse processo mediante as perspectivas do modelo da CIF 31. Logo, a incapacidade em atividades instrumentais de vida diária foi associada em ordem crescente de gravidade aos estágios de fragilidade, corroborando com pesquisas em diferentes populações 1,12. A dependência em uma atividade instrumental gerou uma chance de 1,59 vez de os idosos serem pré-frágeis e de 2,17 vezes de serem frágeis, após controle por fatores de confusão. Isso sugere a predominância dos domínios físicos na síndrome da fragilidade, os quais predizem isoladamente a incapacidade $36,37,38,39$. Dentre os preditores de incapacidade, a redução da velocidade da marcha tem-se destacado em importância e frequência 40 , tendo sido o item do fenótipo mais constante neste estudo, presente em mais de $80 \%$ dos idosos frágeis.

$\mathrm{Na}$ amostra do Município de Belo Horizonte, a fragilidade esteve associada à restrição na participação, demonstrada pela maior chance de redução na realização de atividades avançadas de vida diária, relacionadas à inserção social, cultural, religiosa, política e laboral, mesmo quando controlada por incapacidade em AVD e idade. Tais re- sultados são corroborados pelos de Fairhall et al. 41 que identificaram elevada frequência de restrição à participação em idosos frágeis, com ênfase para a limitação de atividades de laborais.

Os componentes relativos aos fatores pessoais foram representados neste estudo pela associação entre fragilidade e redução da autoeficácia mediante a preocupação com a possibilidade de quedas, que se manteve associada à fragilidade e pré-fragilidade mesmo quando controlada pela ocorrência de quedas, comorbidades e alterações na marcha. Esses dados são suportados pelo estudo de Seematter-Bagnoud et al. 42 que operacionalizou fragilidade segundo Fried et al. 1 e demonstrou forte associação entre baixa autoeficácia e fragilidade, controladas por quedas e desempenho na marcha.

$\mathrm{O}$ ambiente pode atuar como facilitador ou como uma barreira à independência funcional 31. Entretanto, o ambiente hospitalar frequentemente representa um fator negativo em populações de idosos 43,44,45. Na amostra de idosos de Belo Horizonte, a pré-fragilidade e a fragilidade estiveram associadas ao gradiente ascendente na razão de chance para utilização de serviços de saúde incluindo hospitalização, mesmo controlada por fatores de confusão. Os resultados estão de acordo com os de Rochat et al. ${ }^{46}$ que reforçam a forte associação entre fragilidade e uso de serviços de saúde e com os de Gill et al. 43 que confirmaram essa tendência reportando taxas de internação hospitalar crescente entre idosos não frágeis, pré-frágeis e frágeis, respectivamente.

A associação entre fragilidade e queda identificada no modelo desta pesquisa mantém-se após o controle por incapacidade em AVD, comorbidade, limitações na marcha e idade avançada. Esse resultado corrobora os de outros estudos 2,6 e poderia ser influenciado pela associação independente entre queda e os itens dessa síndrome, especialmente a sarcopenia, que isoladamente prediz queda ${ }^{45}$. Alguns pesquisadores destacam a implicação teórica 6,33,43,47 entre fragilidade e queda, relativa à origem multifatorial e complexa de ambas, que transpõem as relações de associação e predição 6,47. Nesse contexto, a queda é incorporada ao modelo de fragilidade como um subciclo dele, podendo desencadear e/ou acelerar essa síndrome, configurando-se como um marcador de falha nesse sistema complexo 33 .

A associação entre fragilidade e comorbidades foi confirmada no atual estudo. Essa associação também foi evidenciada em outro estudo com idosos brasileiros 12 e em pesquisas internacionais 1,48,49. Esse resultado pode ser fundamentado pelo fato de a fragilidade ser determinada por disfunção imunológica, desregulação 
neuroendócrina e processos inflamatórios crônicos 1, assim como pela associação da fragilidade a alterações orgânicas estruturais subclínicas que predispõem os indivíduos a doenças 48 . Existe também uma relação de retroalimentação positiva entre fatores de risco para doenças e fragilidade, fato que incrementa a propensão para desfechos de maior gravidade 48,49.

A sintomatologia depressiva apresentou forte e crescente associação com pré-fragilidade e fragilidade neste estudo, em que os frágeis apresentaram chances 2,6 vezes maiores para sintomas depressivos. Essas chances foram semelhantes entre homens e mulheres e se mantiveram presentes após controle para incapacidades em AVD, comorbidades, satisfação com a vida e autoavaliação de saúde. Esse resultado é reforçado por estudos que identificaram a tendência crescente de sintomas depressivos e níveis de fragilidade 1,50,51. A associação entre fragilidade, depressão e sintomatologia depressiva poderia estar vinculada à sobreposição de características coexistentes em tais condições de saúde, como exemplo, a inatividade, a perda de peso, a exaustão e nível reduzido de atividade física $1,51,52,53$.

Assim, a fragilidade na perspectiva de um modelo complexo se associa a diversos componentes que interagem de forma dinâmica e se constituem simultaneamente em potenciais pontos de entrada ou saída do ciclo de fragilização.

As principais limitações deste estudo poderiam estar vinculadas a eventual possibilidade de coexistirem situações relacionadas à fragilidade que não foram captadas pelo método de operacionalização da condição de saúde utilizada neste trabalho. Nesse contexto, o fenótipo limita a avaliação de possíveis idosos frágeis com alterações cognitivas, restrição de marcha, sequelas motoras graves dentre outras. Outra limitação se constitui na mensuração do item relacionado ao nível de atividade física mensurada pelo MLTPAQ no fenótipo de fragilidade para idosos brasileiros, o qual identifica valores extremamente baixos, podendo indicar algum tipo de inadequação da utilização desse instrumento para tal população.

\section{Conclusão}

Esta pesquisa demonstrou elevada prevalência do fenótipo da fragilidade e identificou fatores associados a essa síndrome passíveis de modificação, uma vez que na presença da fragilidade são maiores as chances para condições adversas de saúde. Destaca-se, portanto, a necessidade de prevenção e intervenção dessa condição de saúde em idosos.

Demosntrou também a ocorrência de um gradiente de associação positiva entre o fenótipo de fragilidade e as condições selecionadas de saúde física, mental e funcional, e de uso de serviços (idade avançada, limitações em AIVD, restrição de AAVD, utilização de dispositivos auxiliares da marcha, maior número de comorbidades, ocorrência de quedas, sintomas depressivos, redução da autoeficácia preventiva de quedas e hospitalização).

Este estudo constatou a associação entre fragilidade e incapacidade com estreita relação entre ambas que ultrapassa a função em AVD. Considerando que o processo de incapacidade pode ser modificado por fatores ambientais, contextuais e intrínsecos ao indivíduo ${ }^{31}$, as condições de fragilidade e especialmente a pré-fragilidade devem ser destacadas na realização de medidas para retardar e atenuar o declínio funcional em idosos, na perspectiva de promoção do envelhecimento ativo. 


\section{Resumen}

El objetivo del estudio fue identificar la prevalencia y los factores asociados a la fragilidad en ancianos que viven en una comunidad de la ciudad de Belo Horizonte, Minas Gerais, Brasil. Se evaluaron los factores clínicos, funcionales y socio-demográficos. Se utilizó un modelo de regresión ordinal, con el fin de verificar las condiciones asociadas con la fragilidad y para determinar el odds ratio $(\alpha=0,05)$. La prevalencia de prefragilidad fue de un 46,3\% y fragilidad un 8,7\%. Los ancianos tipificados como pre-frágiles y frágiles tuvieron, respectivamente, una más alta y creciente proporción de probabilidad de dependencia, respecto a las actividades instrumentales de la vida diaria, restricciones en las actividades avanzadas de la vida diaria, además, se caracterizaban por el uso de dispositivos de asistencia al andar; comorbilidades, caídas, síntomas depresivos, baja auto-eficacia para evitar caídas, hospitalización y edad avanzada. Se identificó un alto porcentaje de los estados de fragilidad, asociada con mayores probabilidades de condiciones adversas para la salud, especialmente, las relacionadas con la incapacidad.

Anciano Frágil; Actividades Cotidianas; Prevalencia

\section{Colaboradores}

R. A. Vieira, R. O. Guerra, A. C. S. Andrade e R. C. Dias participaram da concepção do projeto; análise e interpretação dos dados; redação do artigo; revisão crítica do conteúdo; aprovação final da versão a ser publicada. K. C. Giacomin e K. S. S. Vasconcelos colaboraram na revisão crítica relevante do conteúdo intelectual; aprovação final da versão a ser publicada. L. S. M. Pereira e J. M. D. Dias contribuíram na concepção do projeto e aprovação final da versão a ser publicada.

\section{Agradecimentos}

Ao Proquali-UFJF, CNPq, FAPEMIG e Capes pelo financiamento.

\section{Referências}

1. Fried LP, Tangen CM, Walston J, Newman AB, Hirsch C, Gottdiener J, et al. Frailty in older adults: evidence for a phenotype. J Gerontol A Biol Sci Med Sci 2001; 56:M146-56.

2. Markle-Reid M, Browne G. Conceptualizations of frailty in relation to older adults. J Adv Nurs 2003; 44:58-68.

3. Rockwood K, Mogilner A, Mitnitski A. Changes with age in the distribution of a frailty index. Mech Ageing Dev 2004; 125:517-9.

4. Levers MJ, Estabrooks CA, Ross Kerr JC. Factors contributing to frailty: literature review. J Adv Nurs 2006; 56:282-91.
5. Fried LP, Ferrucci L, Darer J, Williamson JD, Anderson G. Untangling the concepts of disability, frailty, and comorbidity: implications for improved targeting and care. J Gerontol A Biol Sci Med Sci 2004; 59:255-63.

6. Walston JD. Frailty as a model of aging. In: Conn PM, editor. Handbook of models for humon aging. San Diego: Elsevier Academic Press; 2006. p. 697-702.

7. Fried LP, Bandeen-Roche K, Kasper JD, Guralnik JM. Association of comorbidity with disability in older women: the Women's Health and Aging Study. J Clin Epidemiol 1999; 52:27-37. 
8. Lowry KA, Vallejo AN, Studenski SA. Successful aging as a continuum of functional independence: lessons from physical disability models of aging. Aging Dis 2012; 3:5-15.

9. Rockwood K, Howlett SE, MacKnight C, Beattie BL, Bergman H, Hebert R, et al. Prevalence, attributes, and outcomes of fitness and frailty in communitydwelling older adults: report from the Canadian study of health and aging. J Gerontol A Biol Sci Med Sci 2004; 59:1310-7.

10. Santos-Eggimann B, Cuenoud P, Spagnoli J, Junod J. Prevalence of frailty in middle-aged and older community-dwelling Europeans living in 10 countries. J Gerontol A Biol Sci Med Sci 2009; 64:675-81.

11. Alvarado BE, Zunzunegui MV, Beland F, Bamvita JM. Life course social and health conditions linked to frailty in Latin American older men and women. J Gerontol A Biol Sci Med Sci 2008; 63:1399-406.

12. Sousa AC, Dias RC, Maciel AC, Guerra RO. Frailty syndrome and associated factors in communitydwelling elderly in Northeast Brazil. Arch Gerontol Geriatr 2012; 54:e95-101.

13. Brucki SM, Nitrini R, Caramelli P, Bertolucci PH, Okamoto IH. Sugestöes para o uso do mini-exame do estado mental no Brasil. Arq Neuropsiquiatr 2003; 61:777-81.

14. Batistoni SST, Neri AL, Cupertino APFB. Validade da escala de depressão do Center for Epidemiological Studies entre idosos brasileiros. Rev Saúde Pública 2007; 41:598-605.

15. Taylor HL, Jacobs Jr. DR, Schucker B, Knudsen J, Leon AS, DeBacker G. A questionnaire for the assessment of leisure time physical activities. J Chronic Dis 1978; 31:741-55.

16. Lustosa LP, Pereira DS, Dias RC, Parentoni AN, Britto RR, Pereira LSM. Tradução e adaptação transcultural do Minnesota Leisure Time Activities Questionnaire em idosos. Geriatria \& Gerontologia 2011; 5:57-65.

17. Lino VTS, Pereira SRM, Camacho LAB, Ribeiro Filho ST, Buksman S. Adaptação transcultural da Escala de Independência em Atividades da Vida Diária (Escala de Katz). Cad Saúde Pública 2008; 24:103-12.

18. Lawton MP, Brody EM. Assessment of older people: self-maintaining and instrumental activities of daily living. Gerontologist 1969; 9:179-86.

19. Camargos FF, Dias RC, Dias JM, Freire MT. Crosscultural adaptation and evaluation of the psychometric properties of the Falls Efficacy Scale-International Among Elderly Brazilians (FES-I-BRAZIL). Rev Bras Fisioter 2010; 14:237-43.

20. Almeida OP, Almeida SA. Confiabilidade da versão brasileira da Escala de Depressão em Geriatria (GDS) versão reduzida. Arq Neuropsiquiatr 1999; 57:421-6.

21. Abreu MNS, Siqueira AL, Caiaffa WT. Regressão logística ordinal em estudos epidemiológicos. Rev Saúde Pública 2009; 43:183-94.

22. Syddall H, Roberts HC, Evandrou M, Cooper C, Bergman H, Aihie SA. Prevalence and correlates of frailty among community-dwelling older men and women: findings from the Hertfordshire Cohort Study. Age Ageing 2010; 39:197-203.

23. Minayo MCS, Hatz ZMA, Buss PM. Qualidade de vida e saúde: um debate necessário. Ciênc Saúde Coletiva 2000; 5:7-18.
24. Drey M, Pfeifer K, Sieber CC, Bauer JM. The Fried frailty criteria as inclusion criteria for a randomized controlled trial: personal experience and literature review. Gerontology 2011; 57:11-8.

25. Avila-Funes JA, Helmer C, Amieva H, BarbergerGateau P, Le GM, Ritchie K, et al. Frailty among community-dwelling elderly people in France: the three-city study. J Gerontol A Biol Sci Med Sci 2008; 63:1089-96.

26. Ottenbacher KJ, Graham JE, Al SS, Raji M, SamperTernent R, Ostir GV, et al. Mexican Americans and frailty: findings from the Hispanic established populations epidemiologic studies of the elderly. Am J Public Health 2009; 99:673-9.

27. Ostir GV, Ottenbacher KJ, Markides KS. Onset of frailty in older adults and the protective role of positive affect. Psychol Aging 2004; 19:402-8.

28. Espinoza SE, Jung I, Hazuda H. Frailty transitions in the San Antonio Longitudinal Study of Aging. J Am Geriatr Soc 2012; 60:652-60.

29. Xue QL, Bandeen-Roche K, Varadhan R, Zhou J, Fried LP. Initial manifestations of frailty criteria and the development of frailty phenotype in the Women's Health and Aging Study II. J Gerontol A Biol Sci Med Sci 2008; 63:984-90.

30. Xue QL. The frailty syndrome: definition and natural history. Clin Geriatr Med 2011; 27:1-15.

31. Organização Mundial da Saúde. CIF: Classificação Internacional de Funcionalidade, Incapacidade e Saúde. Brasília: Organização Mundial da Saúde; 2003.

32. Yang Y, Lee LC. Dynamics and heterogeneity in the process of human frailty and aging: evidence from the U.S. older adult population. J Gerontol B Psychol Sci Soc Sci 2010; 65B:246-55.

33. Nowak A, Hubbard RE. Falls and frailty: lessons from complex systems. J R Soc Med 2009; 102:98-102.

34. Bateni H, Maki BE. Assistive devices for balance and mobility: benefits, demands, and adverse consequences. Arch Phys Med Rehabil 2005; 86:134-45.

35. Salminen AL, Brandt A, Samuelsson K, Toytari O, Malmivaara A. Mobility devices to promote activity and participation: a systematic review. J Rehabil Med 2009; 41:697-706.

36. Avlund K, Rantanen T, Schroll M. Tiredness and subsequent disability in older adults: The role of walking limitations. J Gerontol A Biol Sci Med Sci 2006; 61:1201-5.

37. Avlund K. Fatigue in older adults: an early indicator of the aging process? Aging Clin Exp Res 2010; 22:100-15

38. Wennie Huang WN, Perera S, VanSwearingen J, Studenski S. Performance measures predict onset of activity of daily living difficulty in communitydwelling older adults. J Am Geriatr Soc 2010; 58: 844-52.

39. Vermeulen J, Neyens JC, van Rossum E, Spreeuwenberg MD, de Witte LP. Predicting ADL disability in community-dwelling elderly people using physical frailty indicators: a systematic review. BMC Geriatr 2011; 11:33. 
40. Abellan van Kan G, Rolland Y, Andrieu S, Bauer J, Beauchet O, Bonnefoy M, et al. Gait speed at usual pace as a predictor of adverse outcomes in community-dwelling older people an International Academy on Nutrition and Aging (IANA) Task Force. J Nutr Health Aging 2009; 13:881-9.

41. Fairhall N, Sherrington C, Kurrle SE, Lord SR, Cameron ID. ICF participation restriction is common in frail, community-dwelling older people: an observational cross-sectional study. Physiotherapy 2011; 97:26-32.

42. Seematter-Bagnoud L, Santos-Eggimann B, Rochat S, Martin E, Karmaniola A, Aminian K, et al. Vulnerability in high-functioning persons aged 65 to 70 years: the importance of the fear factor. Aging Clin Exp Res 2010; 22:212-8.

43. Gill TM, Allore HG, Holford TR, Guo Z. Hospitalization, restricted activity, and the development of disability among older persons. JAMA 2004; 292 2115-24.

44. Gill TM, Allore HG, Gahbauer EA, Murphy TE. Change in disability after hospitalization or restricted activity in older persons. JAMA 2010; 304: 1919-28.

45. Gill TM, Gahbauer EA, Han L, Allore HG. The relationship between intervening hospitalizations and transitions between frailty states. J Gerontol A Biol Sci Med Sci 2011; 66:1238-43.

46. Rochat S, Cumming RG, Blyth F, Creasey H, Handelsman D, Le Couteur DG, et al. Frailty and use of health and community services by communitydwelling older men: the Concord Health and Ageing in Men Project. Age Ageing 2010; 39:228-33.
47. Landi F, Liperoti R, Russo A, Giovannini S, Tosato $\mathrm{M}$, Capoluongo E, et al. Sarcopenia as a risk factor for falls in elderly individuals: results from the ilSIRENTE study. Clin Nutr 2012; 31:652-8.

48. Samper-Ternent R, Karmarkar A, Graham J, Reistetter T, Ottenbacher K. Frailty as a predictor of falls in older Mexican Americans. J Aging Health 2012; 24:641-53.

49. Fulop T, Larbi A, Witkowski JM, McElhaney J, Loeb M, Mitnitski A, et al. Aging, frailty and age-related diseases. Biogerontology 2010; 11:547-63.

50. Weiss CO. Frailty and chronic diseases in older adults. Clin Geriatr Med 2011; 27:39-52.

51. Lakey SL, LaCroix AZ, Gray SL, Borson S, Williams $\mathrm{CD}$, Calhoun D, et al. Antidepressant use, depressive symptoms, and incident frailty in women aged 65 and older from the Women's Health Initiative Observational Study. J Am Geriatr Soc 2012; 60:854-61.

52. Woods NF, LaCroix AZ, Gray SL, Aragaki A, Cochrane BB, Brunner RL, et al. Frailty: emergence and consequences in women aged 65 and older in the women's health initiative observational study. J Am Geriatr Soc 2005; 53:1321-30.

53. Rantanen T, Penninx BW, Masaki K, Lintunen T, Foley D, Guralnik JM. Depressed mood and body mass index as predictors of muscle strength decline in old men. J Am Geriatr Soc 2000; 48:613-7.

Recebido em 28/Mai/2012

Versão final reapresentada em 29/Jan/2013

Aprovado em 05/Mar/2013 
Vieira RA, Guerra RO, Giacomin KC, Vasconcelos KSS, Andrade ACS, Pereira LSM, Dias J MD, Dias RC. Prevalência de fragilidade e fatores associados em idosos comunitários de Belo Horizonte, Minas Gerais, Brasil: dados do Estudo FIBRA. Cad Saúde Pública 2013; 29(8):16311643.

A revista foi informada sobre um erro no primeiro parágrafo da seção Instrumentos e Variáveis. O parágrafo correto é:

The journal has been informed of an error in the first paragraph of the section Instrumentos $e$ Variáveis. The correct paragraph is:

La revista fue informada sobre un error en el primero parágrafo de la sección Instrumentos e Variáveis. El parágrafo correcto es:

\section{Instrumentos e variáveis}

A variável dependente do estudo, síndrome da fragilidade, foi operacionalizada segundo o fenótipo proposto por Fried et al. 1: (1) perda de peso avaliada pelo autorrelato, indicando resposta positiva à perda $\geq \mathrm{a} 4,5 \mathrm{~kg}$ ou $\geq 5 \%$ do peso corporal; (2) exaustão avaliada por duas perguntas da escala de depressão do Center for Epidemiological Studies (CES-D) 14; preenchendo o critério caso ocorresse resposta positiva em pelo menos uma delas; (3) nível de atividade física investigado pelo questionário Minnesota Leisure Time Physical Activity Questionnaire (MLTPAQ) 15,16, apresentado como medida o gasto calórico semanal em quilocalorias (Kcal), ajustado por sexo e estabelecido como pontos de corte o percentil 20 da amostra (idosos abaixo do ponto de corte pontuariam nesse critério); (4) força muscular avaliada pelo teste de força de preensão palmar, ajustado por sexo e quartis do índice de massa corporal (IMC) com pontos de corte determinados pelo percentil 20 da distribuição, utilizando o dinamômetro do tipo Jamar (Sammons Preston, Warrenville, Estados Unidos); (indivíduos abaixo do ponto de corte pontuariam neste critério); (5) tempo de marcha calculado em segundos, medido pelo cronômetro Professional Quartz Timer, modelo KD1O69 (Kadio, China), gasto para percorrer em velocidade habitual, uma distância de 4,6 metros, de um total de 8,6 metros considerando 2,0 metros para aceleração e 2,0 metros para desaceleração da mar- cha (os pontos de corte da amostra foram determinados pelo percentil 80 do tempo, ajustado por sexo e altura; indivíduos acima do ponto de corte preencheriam este critério).

A revista foi informada sobre um erro no segundo parágrafo da seção Resultados. O parágrafo correto é:

The journal has been informed of an error in the second paragraph of the section Resultados. The correct paragraph is:

La revista fue informada sobre un error en el segundo parágrafo de la sección Resultados. El parágrafo correcto es:

A distribuição dos itens positivos para a composição do fenótipo de fragilidade nessa amostra revelou uma proporção de $52(8,7 \%)$ de indivíduos frágeis; 278 (46,3\%) de pré-frágeis e 271 (45,0\%) de idosos não frágeis. 